\title{
Impact of Nonlinear Phase Noise to DPSK Signals: A Comparison of Different Models
}

\author{
Keang-Po Ho, Senior Member, IEEE
}

\begin{abstract}
When a differential phase-shift keying signal is contaminated by nonlinear phase noise, various models to evaluate the error probability are compared. The simplest method is based on the $Q$ factor. The exact method takes into account the dependence between amplifier noise and nonlinear phase noise. All approximated models underestimate both the error probability and the required signal-to-noise ratio.
\end{abstract}

Index Terms-Differential phase-shift keying (DPSK), error analysis, nonlinear wave propagation, phase noise.

\section{INTRODUCTION}

W HEN FIBER loss is periodically compensated by optical amplifiers, the interaction of fiber Kerr effect and amplifier noises induces nonlinear phase noise, often called the Gordon-Mollenauer effect [1] or, more precisely, self-phase modulation induced nonlinear phase noise. Added directly to the signal phase, nonlinear phase noise degrades differential phase-shift keying (DPSK) signals [1]-[7]. Recently, the DPSK signal has received renewed interests for long-haul transmission [4], [8]-[13].

Traditionally, the impact of nonlinear phase noise to DPSK signals is investigated based on the variance or $Q$ factor of the phase [1], [4], [14]-[17]. As a non-Gaussian random variable [3], [7], [18], [19], nonlinear phase noise cannot be completely characterized by either its $Q$ factor or variance. Here, various methods to evaluate the error probability of DPSK signal are compared.

The simplest method is based on the $Q$ factor [16], [17]. When the nonlinear phase noise is approximated as Gaussiandistributed, its statistics is the same as that of laser phase noise and the Nicholson model [20] can be used to evaluate the error probability. Two other methods use the exact model of nonlinear phase noise but with [2], [7], [21] and without [5], [6] taking into account the dependence between amplifier noises and nonlinear phase noise.

With nonlinear phase noise, assuming a zero transmitted phase, the overall received phase is

$$
\Phi_{r}=\Theta_{n}-\Phi_{\mathrm{NL}}=\Theta_{n}-\frac{\left\langle\Phi_{\mathrm{NL}}\right\rangle}{\rho_{s}+\frac{1}{2}} \Phi
$$

where $\Theta_{n}$ is the linear phase noise called the phase of amplifier noise, $\Phi_{\mathrm{NL}}$ is the nonlinear phase noise, $\left\langle\Phi_{\mathrm{NL}}\right\rangle$ is the mean

Manuscript received September 22, 2003; revised January 11, 2004. This work was supported in part by the National Science Council under Grant NSC-92-2218-E-002-034.

The author is with the Institute of Communication Engineering and the Department of Electrical Engineering, National Taiwan University, Taipei 106 Taiwan, R.O.C. (e-mail: kpho@cc.ee.ntu.edu.tw).

Digital Object Identifier 10.1109/LPT.2004.826054 nonlinear phase shift, $\Phi$ is the normalized nonlinear phase noise [7], [19], and $\rho_{s}$ is the signal-to-noise ratio (SNR) defined over an optical matched filter. In (1), the scaled factor of normalization is the ratio of $\left\langle\Phi_{\mathrm{NL}}\right\rangle$ to $\langle\Phi\rangle=\rho_{s}+(1 / 2)$. For the DPSK signal, the differential received phase $\Delta \Phi_{r}$ is the difference of the received phase of (1) in two consecutive symbols, i.e., $\Delta \Phi_{r}=\Phi_{r}(t)-\Phi_{r}(t-T)$ for bit interval of $T$.

\section{GAUSSIAN APPROXIMATION BASED ON $Q$ FACtOR}

The variance of the phase of amplifier noise is [1], [22]

$$
\sigma_{\Theta_{n}}^{2}=\frac{1}{2 \rho_{s}} \text {. }
$$

The variance of nonlinear phase noise is [7], [19]

$$
\sigma_{\Phi_{\mathrm{NL}}}^{2}=\frac{\left\langle\Phi_{\mathrm{NL}}\right\rangle^{2}}{\left(\rho_{s}+\frac{1}{2}\right)^{2}}\left(\frac{2}{3} \rho_{s}+\frac{1}{6}\right) \approx \frac{2\left\langle\Phi_{\mathrm{NL}}\right\rangle^{2}}{3 \rho_{s}} .
$$

The approximation in (3) was given in [1]. Using both (2) and (3), for the DPSK signal, the $Q$ factor is [16]

$$
Q=\frac{\pi}{2 \sqrt{2\left(\sigma_{\Theta_{n}}^{2}+\sigma_{\Phi_{\mathrm{NL}}}^{2}\right)}}
$$

where $\pi / 2$ is the phase difference between the constellation points and the decision threshold, and a further factor of two is for the differential signal. Based on the $Q$ factor, the error probability is $p_{e}=(1 / 2) \operatorname{erfc}(Q / \sqrt{2})$, where $\operatorname{erfc}(\cdot)$ is the complementary error function.

\section{Gaussian APPROXimation OF NONLINEAR Phase NoISE (NICHOLSON MODEL)}

The phase of amplifier noise of $\Theta_{n}$ is certainly non-Gaussian distributed [22]. The assumption of Gaussian distribution of the phase underestimates the error probability by $\pi / 2$ as an SNR gain for phase-shift keying (PSK) signal. When the nonlinear phase noise difference between two consecutive symbols is assumed to be Gaussian distributed, the variance of $2 \sigma_{\Phi_{\mathrm{NL}}}^{2}$ is sufficient to characterize the nonlinear phase noise. Similar to that of DPSK signals with laser phase noise [6], [20], the error probability is

$$
\begin{aligned}
p_{e}= & \frac{1}{2}-\frac{\rho_{s} e^{-\rho_{s}}}{2} \\
& \cdot \sum_{k=0}^{\infty} \frac{(-1)^{k}}{2 k+1}\left[I_{k}\left(\frac{\rho_{s}}{2}\right)+I_{k+1}\left(\frac{\rho_{s}}{2}\right)\right]^{2} \\
& \times \exp \left[-(2 k+1)^{2} \sigma_{\Phi_{\mathrm{NL}}}^{2}\right]
\end{aligned}
$$

where $I_{n}(\cdot)$ is the $n$ th-order modified Bessel function of the first kind. 
In (5), the term of $\exp \left[-(2 k+1)^{2} \sigma_{\Phi_{N I}}^{2}\right]$ is the characteristic function of the Gaussian distributed phase noise at "angular frequency" of $2 k+1$. When the laser phase noise is modeled as a Wiener process, the phase noise difference for a time interval of $T$ is a Gaussian-distributed random variable having a variance of $2 \pi \Delta \nu T$ for a laser linewidth of $\Delta \nu$ [20].

\section{INDEPENDENCE APPROXIMATION OF NON-GAUSSIAN NONLINEAR PHASE NOISE}

The characteristic function of the normalized nonlinear phase noise is [7], [19]

$$
\Psi_{\Phi}(\nu)=\sec \sqrt{j \nu} \exp \left[\rho_{s} \sqrt{j \nu} \tan \sqrt{j \nu}\right]
$$

where $\nu$ is the "angular frequency" in Fourier transform to get the characteristic function.

Replacing the Gaussian characteristic function in (5) by the characteristic function of (6), we get

$$
\begin{aligned}
p_{e}= & \frac{1}{2}-\frac{\rho_{s} e^{-\rho_{s}}}{2} \\
& \cdot \sum_{k=0}^{\infty} \frac{(-1)^{k}}{2 k+1}\left[I_{k}\left(\frac{\rho_{s}}{2}\right)+I_{k+1}\left(\frac{\rho_{s}}{2}\right)\right]^{2} \\
& \times\left|\Psi_{\Phi}\left[\frac{(2 k+1)\left\langle\Phi_{\mathrm{NL}}\right\rangle}{\rho_{s}+\frac{1}{2}}\right]\right|^{2} .
\end{aligned}
$$

While exact models are used for both nonlinear phase noise and the phase of amplifier noise separately, the error probability of (7) assumes that nonlinear phase noise is independent of the phase of amplifier noise $\Theta_{n}$. Similar to (5) or [20], the error probability of (7) is similar to the cases [23]-[25] when the additive phase noise is independent of the amplifier noise.

\section{EXACT MOdEL}

The phase of amplifier noise $\Theta_{n}$ is uncorrelated with the nonlinear phase noise, i.e., $E\left\{\Theta_{n} \Phi_{\mathrm{NL}}\right\}=0$. As non-Gaussian random variables, they weakly depend on each other. When the dependence between the phase of amplifier noise and nonlinear phase noise is taken into account, the error probability is [7], [21]

$$
\begin{aligned}
p_{e}= & \frac{1}{2}-\frac{1}{2} \sum_{k=0}^{\infty} \frac{(-1)^{k}\left|\lambda_{k} e^{-\lambda_{k}}\right|}{2 k+1}\left|I_{k}\left(\frac{\lambda_{k}}{2}\right)+I_{k+1}\left(\frac{\lambda_{k}}{2}\right)\right|^{2} \\
& \times\left|\Psi_{\Phi}\left[\frac{(2 k+1)\left\langle\Phi_{\mathrm{NL}}\right\rangle}{\rho_{s}+\frac{1}{2}}\right]\right|^{2}
\end{aligned}
$$

where

$$
\lambda_{k}=\frac{2\left[\frac{j(2 k+1)\left\langle\Phi_{\mathrm{NL}}\right\rangle}{\rho_{s}+\frac{1}{2}}\right]^{1 / 2}}{\sin \left\{2\left[\frac{j(2 k+1)\left\langle\Phi_{\mathrm{NL}}\right\rangle}{\rho_{s}+\frac{1}{2}}\right]^{1 / 2}\right\}} \rho_{s}, \quad k \geq 0
$$

are equivalent to the "angular frequency" depending SNR parameters. Error probability similar to (8) was derived by Mecozzi [2] but for the PSK signal. The exact model of (8) makes no approximation to either the nonlinear phase noise or the phase of amplifier noise of (1), even taking into account their dependence.

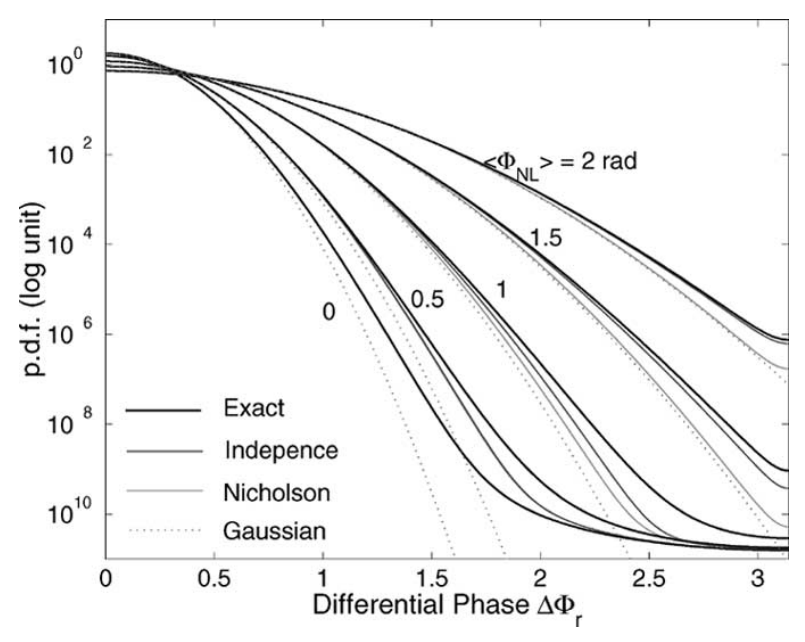

Fig. 1. Probability density of differential phase $\Delta \Phi_{r}$.

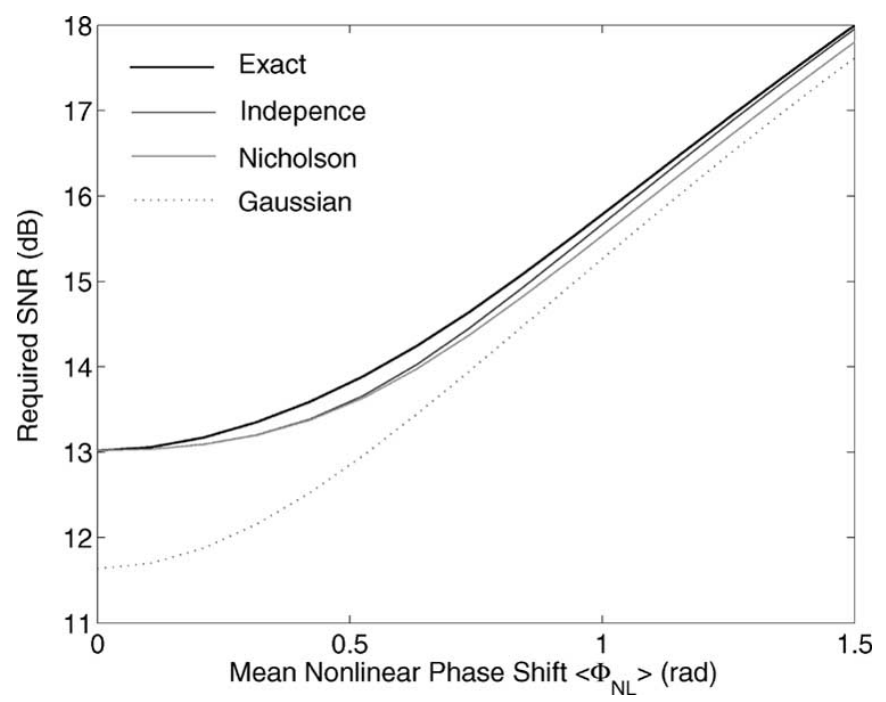

Fig. 2. Required SNR of DPSK signal as a function of mean nonlinear phase shift $\left\langle\Phi_{N L}\right\rangle$.

Because the magnitude of $\left|\lambda_{k}\right| \leq \rho_{s}$, i.e., smaller equivalent SNR, the error probability of (8) is larger than the independence assumption of (7).

\section{NUMERICAL RESULTS}

Fig. 1 shows the probability density function (pdf) of the differential phase for the DPSK signal according to different models. In Fig. 1, the transmitted phases in two consecutive timing intervals are assumed to be the same. Because the pdf is symmetrical with respect to zero, only the pdf from $[0, \pi]$ is shown in Fig. 1. From Fig. 1, all approximated models underestimate the spreading of the differential phase. Decreasing fast, the Gaussian approximation gives a very small probability density at the tail, especially for small mean nonlinear phase shift $\left\langle\Phi_{\mathrm{NL}}\right\rangle$. With smaller pdf spreading than the exact model, all approximated models underestimate the error probability of DPSK signals with nonlinear phase noise.

Fig. 2 shows the required SNR for an error probability of $10^{-9}$ as a function of mean nonlinear phase shift $\left\langle\Phi_{\mathrm{NL}}\right\rangle$. From Fig. 2, all approximated models underestimate the required 
TABLE I

DifFERENT Models FOR DPSK Signal With NoNLINEAR PHASE NOISE

\begin{tabular}{l|l|l|c|c|c}
\hline Model & $\Theta_{n}$ & $\Phi_{\mathrm{NL}}$ & Dependence & $\begin{array}{c}1 \mathrm{~dB} \\
\text { Penalty }\end{array}$ & $\begin{array}{c}\text { Optimal } \\
\text { Point }\end{array}$ \\
\hline $\begin{array}{l}\text { Gaussian [1], [14], [16], [17] } \\
\text { Nicholson [20] }\end{array}$ & Gaussian & Gaussian & Ind. & $0.45 \mathrm{rad}$ & $0.86 \mathrm{rad}$ \\
Independent [5], [6] & non-Gaussian & Gaussian & Ind. & $0.64 \mathrm{rad}$ & $0.95 \mathrm{rad}$ \\
Exact [2], [7], [21] & non-Gaussian & non-Gaussian & Ind. & $0.63 \mathrm{rad}$ & $0.92 \mathrm{rad}$ \\
\hline
\end{tabular}

SNR. Table I summarizes the key parameters from various models. The optimal operating point is such that the increase of mean nonlinear phase shift, proportional to SNR, is larger than the increase of required SNR. The Nicholson and independence approximation give larger (about 13\%) mean nonlinear phase shift for 1-dB power penalty but smaller (within 6\%) optimal operating point than the exact model.

While the error probability based on $Q$ factor is not able to predict the system performance except for the system with large nonlinear phase noise, the Nicholson [20] and independence [5], [6] approximation of nonlinear phase noise underestimate the required SNR of up to 0.27 and $0.23 \mathrm{~dB}$, respectively, and may not conform to the principle of conservative system design. If a prior correction of about $0.3 \mathrm{~dB}$ is added to both the Nicholson and independence approximation, both models can provide a conservative system design.

\section{CONCLUSION}

Various models to evaluate the error probability of DPSK signals with nonlinear phase noise have been compared with each other. All approximated models underestimate both the error probability and required SNR. Although both the Nicholson and independence models give an error probability close to the exact model, both models do not conform to the principle of conservative system design.

\section{REFERENCES}

[1] J. P. Gordon and L. F. Mollenauer, "Phase noise in photonic communications systems using linear amplifiers," Opt. Lett., vol. 15, no. 23, pp. $1351-1353,1990$.

[2] A. Mecozzi, "Limits to long-haul coherent transmission set by the Kerr nonlinearity and noise of the in-line amplifiers," J. Lightwave Technol., vol. 12, pp. 1993-2000, Nov. 1994.

[3] H. Kim and A. H. Gnauck, "Experimental investigation of the performance limitation of DPSK systems due to nonlinear phase noise," IEEE Photon. Technol. Lett., vol. 15, pp. 320-322, Feb. 2003.

[4] C. Xu, X. Liu, L. F. Mollenauer, and X. Wei, "Comparison of return-to-zero differential phase-shift keying and on-off keying long-haul dispersion managed transmission," IEEE Photon. Technol. Lett., vol. 15, pp. 617-619, Apr. 2003.

[5] K.-P. Ho, "Performance degradation of phase-modulated systems due to nonlinear phase noise," IEEE Photon. Technol. Lett., vol. 15, pp. 1213-1215, Sept. 2003.

[6] — - "Compensation improvement of DPSK signal with nonlinear phase noise," IEEE Photon. Technol. Lett., vol. 15, pp. 1216-1218, Sept. 2003.
[7] _- "Statistical properties of nonlinear phase noise," in Advances in Optics and Laser Research, W. T. Arkin, Ed. Commack, NY: Nova, 2003, vol. 3.

[8] A. H. Gnauck, G. Raybon, S. Chandrasekhar, J. Leuthold, C. Doerr, L. Stulz, A. Agrawal, S. Banerjee, D. Grosz, S. Hunsche, A. Kung, A. Marhelyuk, D. Maymar, M. Movassaghi, X. Liu, C. Xu, X. Wei, and D. M. Gill, " $2.5 \mathrm{~Tb} / \mathrm{s}(64 \times 42.7 \mathrm{~Gb} / \mathrm{s})$ transmission over $40 \times 100 \mathrm{~km}$ NZDSF using RZ-DPSK format and all-Raman-amplified spans," in Tech. Dig. Optical Fiber Communication Conf., Anaheim, CA, 2002, Postdeadline Paper FC2.

[9] R. A. Griffin, R. I. Johnstone, R. G. Walker, J. Hall, S. D. Wadsworth, K. Berry, A. C. Carter, M. J. Wale, P. A. Jerram, and N. J. Parsons, "10 $\mathrm{Gb} / \mathrm{s}$ optical differential quadrature phase shift key (DQPSK) transmission using GaAs/AlGaAs integration," in Tech. Dig. Optical Fiber Communication Conf., Anaheim, CA, 2002, Postdeadline Paper FD6.

[10] A. H. Gnauck, G. Raybon, S. Chandrasekhar, J. Leuthold, C. Doerr, L. Stulz, and E. Burrows, " $25 \times 40-\mathrm{Gb} / \mathrm{s}$ copolarized DPSK transmission over $12 \times 100-\mathrm{km}$ NZDF with 50-GHz channel spacing," IEEE Photon. Technol. Lett., vol. 15, pp. 467-469, Mar. 2003.

[11] J.-X. Cai, D. G. Foursa, C. R. Davidson, Y. Cai, G. Domagala, H. Li, L. Liu, W. W. Patterson, A. N. Pilipetskii, M. Nissov, and N. S. Bergano, "A DWDM demonstration of $3.73 \mathrm{~Tb} / \mathrm{s}$ over $11000 \mathrm{~km}$ using 373 RZ-DPSK channels at $10 \mathrm{~Gb} / \mathrm{s}$," in Tech. Dig. Optical Fiber Communication Conf., Atlanta, GA, 2003, Postdeadline Paper PD22.

[12] C. Rasmussen, T. Fjelde, J. Bennike, F. Liu, S. Dey, B. Mikkelsen, P. Mamyshev, P. Serbe, P. van de Wagt, Y. Akasaka, D. Harris, D. Gapontsev, V. Ivshin, and P. Reeves-Hall, "DWDM 40 G transmission over trans-Pacific distance $(10,000 \mathrm{~km})$ using CSRZ-DPSK, enhanced FEC and all-Raman amplified $100 \mathrm{~km}$ Ultra-Wave fiber spans," in Tech. Dig. Optical Fiber Communication Conf., Atlanta, GA, 2003, Postdeadline Paper PD18.

[13] B. Zhu, L. E. Nelson, S. Stulz, A. H. Gnauck, C. Doerr, J. Leuthold, L. Grüner-Nielsen, M. O. Pederson, J. Kim, R. Lingle, Y. Emori, Y. Ohki, N. Tsukiji, A. Oguri, and S. Namiki, "6.4-Tb/s $(160 \times 42.7 \mathrm{~Gb} / \mathrm{s})$ transmission with $0.8 \mathrm{bit} / \mathrm{s} / \mathrm{Hz}$ spectral efficiency over $32 \times 100 \mathrm{~km}$ of fiber using CSRZ-DPSK format," in Tech. Dig. Optical Fiber Communication Conf., Atlanta, GA, 2003, Postdeadline Paper PD19.

[14] X. Liu, X. Wei, R. E. Slusher, and C. J. McKinstrie, "Improving transmission performance in differential phase-shift-keyed systems by use of lumped nonlinear phase-shift compensation," Opt. Lett., vol. 27, no. 18, pp. 1616-1618, 2002.

[15] C. Xu and X. Liu, "Postnonlinearity compensation with data-driven phase modulators in phase-shift keying transmission," Opt. Lett., vol. 27, no. 18, pp. 1619-1621, 2002.

[16] X. Wei, X. Liu, and C. Xu, $Q$ Factor in Numerical Simulations of DPSK with Optical Delay Demodulation, unpublished e-print manuscript no. physics/0 304002.

[17] _ "Numerical simulation of the SPM penalty in a 10-Gb/s RZ-DPSK system," IEEE Photon. Technol. Lett., vol. 15, pp. 1636-1638, Nov. 2003.

[18] K.-P. Ho, "Probability density of nonlinear phase noise," J. Opt. Soc. Amer. B, vol. 20, no. 9, pp. 1875-1879, 2003.

[19] - "Asymptotic probability density of nonlinear phase noise," Opt. Lett., vol. 28, no. 15, pp. 1350-1352, 2003.

[20] G. Nicholson, "Probability of error for optical heterodyne DPSK system with quantum phase noise," Electron. Lett., vol. 20, no. 24, pp. 1005-1007, 1984.

[21] K.-P. Ho, "Exact error probability of phase-modulated signals with nonlinear phase noise," J. Lightwave Technol., submitted for publication.

[22] J. G. Proakis, Digital Communications, 4th ed. New York: McGraw Hill, 2000, sec. 5.2.

[23] N. M. Blachman, "The effect of phase error on DPSK error probability," IEEE Trans. Commun., vol. COM-29, pp. 364-465, Mar. 1981.

[24] P. C. Jain, "Error probabilities in binary angle modulation," IEEE Trans. Inform. Theory, vol. IT-20, pp. 36-42, Jan. 1974.

[25] G. Jacobsen and I. Garrett, "Theory for optical heterodyne DPSK receivers with post-detection filtering," J. Lightwave Technol., vol. 5, pp. 478-484, Apr. 1987. 